Immunohistochemical studies demonstrated that PSCA is expressed mainly in the isthmus of the gastric epithelium, presumably in both precursor and differentiated cells, but that the gene is downregulated in gastric cancer tissue, especially the diffuse type. Transfection studies in gastric cancer cell lines indicated that PSCA inhibits cell proliferation. In a reporter assay, replacement of the $\mathrm{C}$ allele with the risk allele $\mathrm{T}$ decreased the transcriptional activity of PSCA. Even though the function of PSCA is unknown, the authors suggest that the gene might be involved in regulating gastric epithelial-cell proliferation.

Original article The Study Group of Millennium Genome Project for Cancer (2008) Genetic variation in PSCA is associated with susceptibility to diffuse-type gastric cancer. Nat Gen 40: 730-740

\section{Common single nucleotide polymorphisms are linked to neuroblastoma}

Little is known about the factors that lead to neuroblastoma, and previous epidemiological and genetic studies have found no common environmental factor or genetic mutation associated with the disease. Maris et al. investigated the hypothesis that multiple, common DNA variations might predispose an individual to neuroblastoma.

A genome-wide association study was conducted on blood DNA samples from 1,032 children with newly diagnosed neuroblastoma who were registered with the Children's Oncology Group and from 2,043 children with no serious medical disorder who were recruited through the Children's Hospital of Philadelphia Health Care Network. All children were of European ancestry. The genome-wide analysis identified three single nucleotide polymorphisms at chromosome 6p22 that were significantly associated with neuroblastoma (allelic odds ratio $1.39-1.40 ; P=1.71 \times 10^{-9}-7.01 \times 10^{-10}$ ). These correlations remained significant after correction for population substructure by use of Eigenstrat analysis $\left(P=5.82 \times 10^{-8}\right.$ $\left.-8.54 \times 10^{-8}\right)$.

When the associations were tested in a second cohort of children (720 with neuroblastoma and 2,128 healthy controls), the three high-risk alleles were found to be over-represented in the neuroblastoma group. Patients who were homozygous for the high-risk alleles were more likely to have stage IV disease, MYCN oncogene amplification and be classified as highrisk. Furthermore, homozygosity for the high-risk allele of the most significantly associated single nucleotide polymorphism resulted in an almost twofold increase in the risk of developing neuroblastoma (odds ratio 1.97).

The authors note that studies investigating the contribution of these common variants to the development of neuroblastoma are currently being conducted.

Original article Maris JM et al. (2008) Chromosome 6p22 locus associated with clinically aggressive neuroblastoma. N Engl J Med 358: 2585-2593

\section{Increased c-kit expression predicts poor outcome in acute myeloid leukemia}

The importance of c-kit in acute myeloid leukemia (AML) has prompted investigations to assess whether there is a correlation between expression of this protein and prognosis of the disease. Studies have shown conflicting results, however, which might partly be due to differences in methods used to measure c-kit expression. Advani et al. used mean fluorescent index (MFI), a quantitative and reproducible measurement obtained by flow cytometry, to show that c-kit expression is inversely related to survival in AML.

In this retrospective study, bone marrow or blood samples were analyzed from 152 patients newly diagnosed with $A M L$ at the Cleveland Clinic during the period 2000-2005. After these samples were analysed by flow cytometry and the blasts were isolated, MFI was calculated as the mean fluorescence of the blasts divided by the mean autofluorescence of unstained cells; increased MFI values correspond to increased c-kit expression. In multivariate analysis, an MFI of $>20.3$ was significantly associated with decreased overall and progression-free survival (hazard ratio 1.60, $P=0.027$, and hazard ratio $1.67, P=0.039$, respectively). This association was independent of age, white blood cell count at diagnosis, and cytogenetics.

The authors suggest that any clinic with a flow scattergram could calculate MFI and thereby estimate survival on the basis of c-kit expression in patients with AML. They add that 